\section{Measurement of urinary constituents and output using disposable napkins}

Sir,

Drs Roberts and Lucas ${ }^{1}$ have produced an excellent and carefully conducted study that should give more confidence to those performing metabolic studies on babies. I would issue a note of caution, however, to any considering using this method in the premature newborn.

Low birthweight neonates often have irregular, infrequent, and incomplete bladder emptying and may pass only 0.5 or $1 \mathrm{ml}$ at a time. Urine volume measurements based on weighing cotton wool balls or even nappies will be imprecise, more so because in our experience there is considerable evaporation, even inside an enclosed, humidified incubator. Furthermore, for the same reason, the urine samples from successive micturitions cannot be pooled, so that measurement of excretion of urine constituents over a period long enough to avoid inaccuracy due to incomplete bladder emptying will depend on multiple estimations on several samples, making the procedure cumbersome.

B H WILKINS
Southmead Hospital,
Bristol,
England

Drs Roberts and Lucas comment:

Dr Wilkins is mistaken in his suggestion that our new method for measuring urine output in infants ${ }^{1}$ is not suitable for use in low birthweight infants. The problems he describes, infrequent and incomplete bladder emptying, apply equally to the traditional method of using a urine bag and, in any case, are irrelevant to methodological precision as the method is designed to measure urine output not urine synthesis. A separate consideration is the precision of the balance used to weigh the napkins-it should, of course, be related to the amount of urine being measured (a sample of $0.5-1.0 \mathrm{~g}$ requires a precision of at least $\pm 0.01 \mathrm{~g}$ ). Dr Wilkins also suggests that error may be incurred by the urine evaporating from the napkins. Part of our study, however, was designed to measure the rate of evaporation, and the value obtained was reported (less than $0.4 \%$ per hour in a close fitting napkin). We suggest that any problem he has experienced with evaporation may well be due to a lack of care in positioning the napkins. His final point, that the method is cumbersome, is also incorrect; weighing napkins and pooling samples for laboratory analysis are extremely simple procedures compared with trying to keep a urine bag on an infant (boy or girl).

\section{Reference}

1 Roberts SB, Lucas A. Measurement of urinary constituents and output using disposable napkins. Arch Dis Child 1985;60: $1021-4$.

\section{... And children first?}

Sir,

I am the convenor of the British Paediatric Association/ British Association of Paediatric Surgeons Accident Committee. I can assure Dr Choonara' ${ }^{1}$ that we are working actively both as a committee and in close association with the Child Accident Prevention Trust (CAPT) to save children's lives and to improve the quality of their lives by reducing accidents to children.

The CAPT has set up a working party of which I am a $\vec{\circ}$ member to look at what can be done to improve the safety of children in cars and to make recommendations about $\vec{\omega}$ how these can best be implemented.

Examples of topics with which the Accident Committee is currently involved are to press for the safer packaging of drugs to reduce the dangers to young children and to discuss with the toy manufacturers the possibility of making small plastic toys radio-opaque so that in the event $N$ of their being inhaled or swallowed they are easier to identify and remove. There are many others.

The previous convenor of the Accident Committee, Dr $\stackrel{\circ}{3}$ Hugh Jackson, is the medical advisor of the CAPT and has been responsible for many valuable papers and seminars $T$ about hazards to children and has done a great deal to $\underline{0}$ promote interest and involvement of other professionalsfor example, architects, town planners, etc.

We would like more paediatricians to be interested in this subject.

Reference
'Choonara IA. . . . And children first? (letter). Arch Dis Chitu
1985;60:1108.
C ILLINGworTH
Sheffield,
England

\section{Injections and Asian children}

Sir,

Asian children, particularly between the ages of 1 and 5 years, tend to be more difficult to examine than an English child of similar age. In most Asian homes a child is often threatened with being taken to the doctor and given an injection if he does not behave.

In the outpatient situation those of us who understand the Asian language often hear mum say that 'he is not going to give you an injection'. At this the child, who may have been quite cooperative up to that point, bursts out crying and becomes difficult to examine at the very mention of the word 'injection'. This, I feel, is one of the reasons why many Asian parents fail to keep their child's appointment. To make our task and that of the general practitioner easier, community doctors and health visitors should positively discourage this habit of threatening the child at home.

A Rashid Gatrad Birmingham University, Birmingham England 